\title{
The Hamilton-Jacobi Equation, Then and Now
}

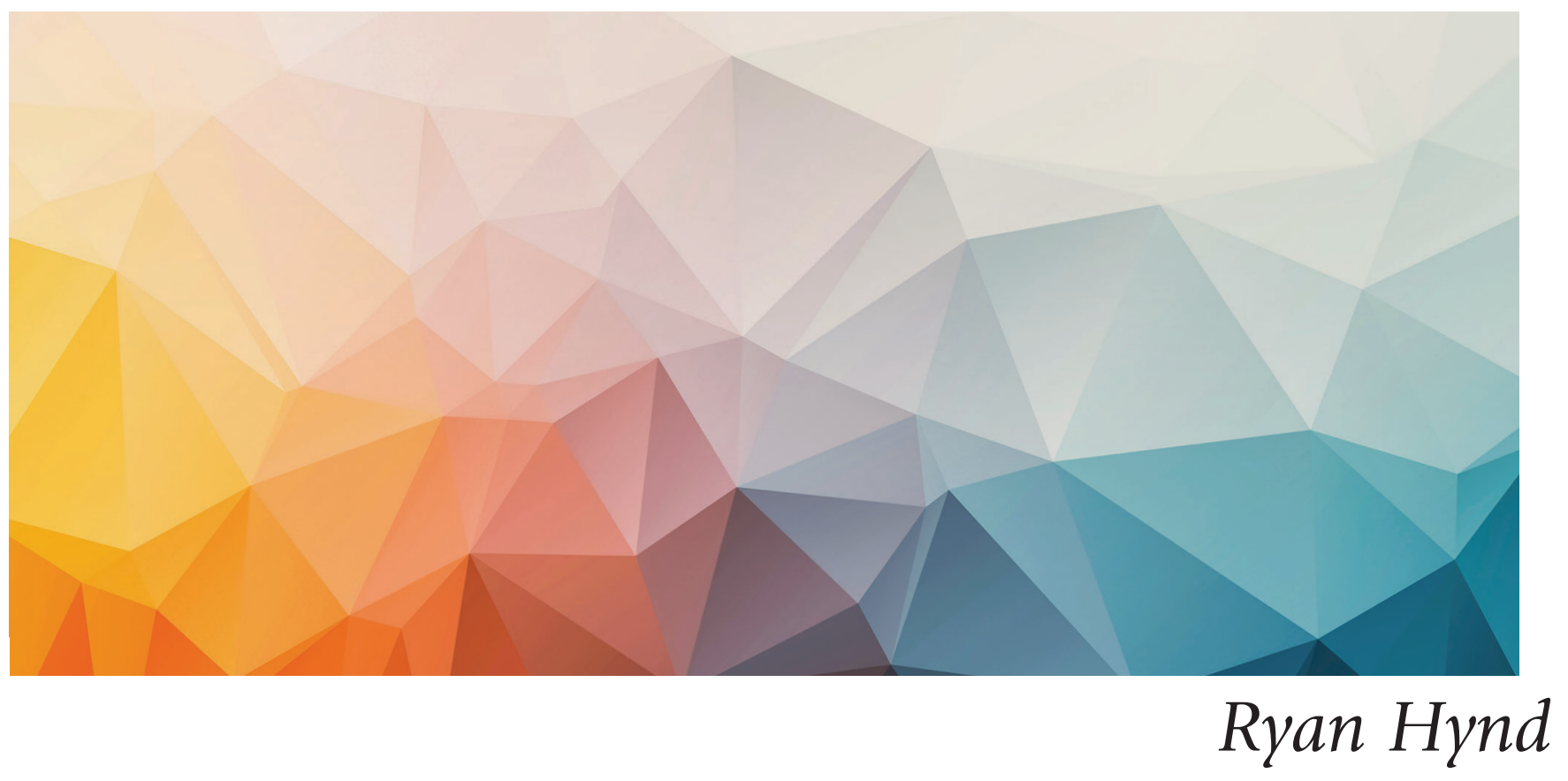

\section{Introduction}

The story of the Hamilton-Jacobi equation begins in classical mechanics as developed in the 19th century. A basic problem within this theory is to describe the motion of a particle with mass $m$ subject to a force $F$ depending on its position. If this particle happens to be moving along the $x$-axis, Newton's second law takes the form

$$
m \ddot{\gamma}(s)=F(\gamma(s)) .
$$

Here $s$ denotes time, $x=\gamma(s)$ is the position of the particle at time $s$, and - is differentiation with respect to time. Observe that in order to determine the trajectory of the particle, we are left to solve a differential equation for given initial conditions $\gamma(0)$ and $\dot{\gamma}(0)$.

An interesting thing happens when we select a potential energy function $V$ for which

$$
F=-V^{\prime}
$$

Ryan Hynd is an associate professor of mathematics at the University of Pennsylvania. His email address is rhynd@math. upenn.edu.

Communicated by Notices Associate Editor Daniela De Silva.

For permission to reprint this article, please contact:

reprint-permission@ams.org.

DOI: https://doi.org/10.1090/noti2352

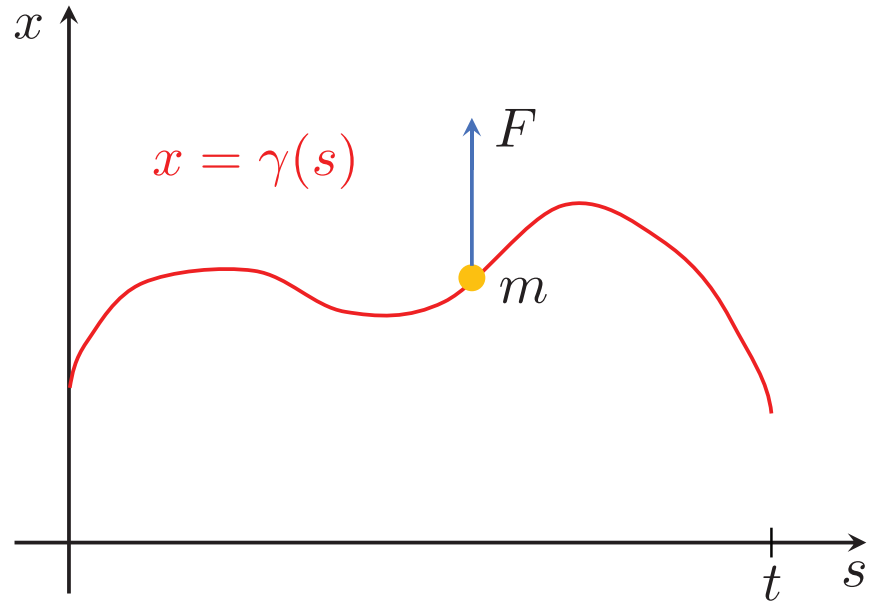

Figure 1. The position $x=\gamma(s)$ of a point mass $m$ versus time on the interval $s \in[0, t]$. This particle moves along the $x$-axis and is subject to a force $F$. We can find $\gamma$ by solving the differential equation (1).

This choice allows us to derive the differential equation (1) through a least action principle. Indeed we may consider the action integral

$$
\mathcal{S}_{t}(\zeta):=\int_{0}^{t} \frac{m}{2} \dot{\zeta}(s)^{2}-V(\zeta(s)) d s
$$


for a given path $\zeta:[0, t] \rightarrow \mathbb{R}$; here and throughout, all paths are assumed to be absolutely continuous. If there is a path $\gamma$ such that

$$
\mathcal{S}_{t}(\gamma) \leq \mathcal{S}_{t}(\zeta)
$$

for each path $\zeta$ with $\zeta(t)=\gamma(t)$, then $\gamma$ solves the Newton's second law differential equation

$$
m \ddot{\gamma}(s)=-V^{\prime}(\gamma(s))
$$

for $s \in(0, t)$ along with

$$
\dot{\gamma}(0)=0 .
$$

Therefore, action-minimizing paths are natural candidates to describe the motion of a particle with mass $m$ subject to a force $-V^{\prime}$.

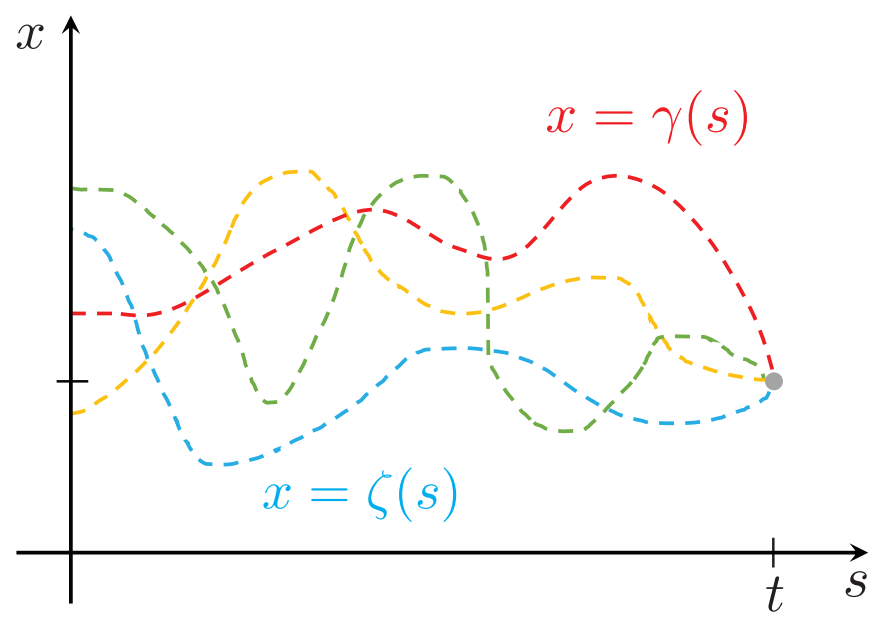

Figure 2. Various trajectories $\zeta:[0, t] \rightarrow \mathbb{R}$ with the same right endpoint as $\gamma$ indicated with the gray dot. All of these trajectories are candidates to minimize the action $\mathcal{S}_{t}$ subject to this right endpoint constraint.

The action integral $\mathcal{S}_{t}$ is also known to satisfy a partial differential equation (PDE). Assume that for each $x \in \mathbb{R}$ and $t>0$,

$$
\gamma^{x, t}:[0, t] \rightarrow \mathbb{R}
$$

is a path which minimizes $\mathcal{S}_{t}(\zeta)$ among all paths $\zeta$ which fulfill the right endpoint constraint $\zeta(t)=x$. Further suppose that when the action integral is evaluated along $\gamma^{x, t}$,

$$
u(x, t)=\mathcal{S}_{t}\left(\gamma^{x, t}\right),
$$

it is a smooth function. Then direct computation (as explained in lecture 19 of [Jac84]) shows that $u$ solves what is now known as a Hamilton-Jacobi equation

$$
\partial_{t} u+\frac{1}{2 m}\left(\partial_{x} u\right)^{2}+V(x)=0 .
$$

Here $\partial_{t} u$ and $\partial_{x} u$ denote partial differentiation with respect to $t$ and $x$, respectively.

In this article, we will examine some of the mathematical developments concerning Hamilton-Jacobi equations since the mid-20th century. This includes the advent of viscosity solutions, which gives us a way to interpret how functions like $u$ defined above generally solve HamiltonJacobi equations. Another key idea we will discuss is the dynamic programming principle from control theory. In addition, we will present research directions involving interacting systems of particles and noncooperative differential games which aim to further expand what we know about Hamilton-Jacobi type equations.

\section{Modeling Applications}

Before studying the particular equation (3), we will discuss various optimization problems in which Hamilton-Jacobi equations arise. We will not analyze each of the resulting equations separately nor present a theory which encompasses them all. However, the ideas that we will subsequently introduce for equation (3) can be adapted to all of these equations. The purpose of this section is to give some perspective on the applicability of these concepts. We also note that some of the PDEs in the examples below are typically called Hamilton-Jacobi-Bellman equations, as they can be derived with Bellman's dynamic programming method which we will discuss later in this article.

2.1. Escape of a light ray. Let us first consider a twodimensional light ray passing through an inhomogeneous medium. We will represent the medium by a bounded open subset $U$ of the $x y$-plane and the light ray by a path

$$
\gamma:[0, \tau] \rightarrow U ; t \mapsto\left(\gamma^{x}(t), \gamma^{y}(t)\right) .
$$

We will also assume that at each point $(x, y)$ in $U$, the inhomogeneity of the medium constrains the speed of light to be no more than $c(x, y)$ for a given positive function $c$.

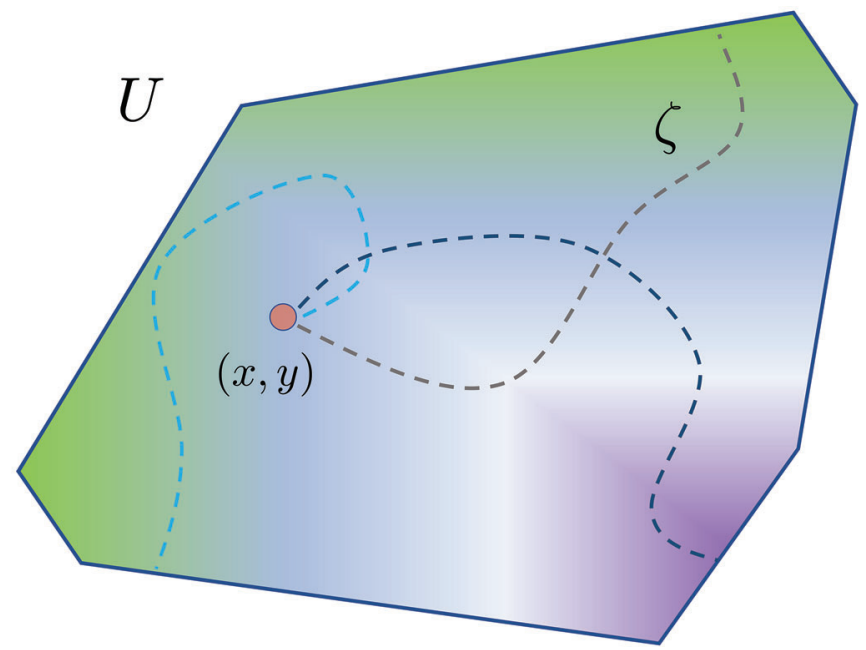

Figure 3. A planar domain $U$ which represents an inhomogeneous medium. The dashed curves display possible light ray paths $\zeta:\left[0, \tau_{\zeta}\right] \rightarrow U$ emanating from a common point $\zeta(0)=(x, y)$ in $U$. Fermat's principle tells us that light ray paths $\gamma$ are among those which minimize their exit time $\tau_{\gamma}$ from $U$. 
For any path $\zeta:[0, \infty) \rightarrow \mathbb{R}^{2}$ with $\zeta(0)$ in $U$, we define

$$
\tau_{\zeta}:=\min \{t \geq 0: \zeta(t) \notin U\}
$$

as the first time that $\zeta$ exits $U$ and $\tau_{\zeta}=\infty$ if no such time exists. According to Fermat's principle, a ray of light assumes a path which takes the least amount of time to exit this region. As a result we will try to find a path $\zeta$ which satisfies the speed constraint

$$
|\dot{\zeta}(t)|:=\sqrt{\left(\dot{\zeta}^{x}(t)\right)^{2}+\left(\dot{\zeta}^{y}(t)\right)^{2}} \leq c(\zeta(t))
$$

and minimizes $\tau_{\zeta}$. To this end, we consider

$$
u(x, y)=\min \left\{\tau_{\zeta}: \zeta(0)=(x, y),|\dot{\zeta}(t)| \leq c(\zeta(t))\right\}
$$

for $(x, y)$ in $U$.

It turns out that $u$ can be interpreted as a solution of the eikonal equation

$$
c(x, y) \sqrt{\left(\partial_{x} u\right)^{2}+\left(\partial_{y} u\right)^{2}}=1 .
$$

This PDE may be the first Hamilton-Jacobi equation ever written down [Ham28]. An important example occurs when

$$
c(x, y)=1 \text { for all }(x, y) \text { in } U .
$$

This corresponds to a homogeneous medium. Here $u(x, y)$ is simply the distance from $(x, y)$ to the boundary of $U$. In particular, the distance to the boundary function is a solution of the PDE

$$
\sqrt{\left(\partial_{x} u\right)^{2}+\left(\partial_{y} u\right)^{2}}=1
$$

We recommend [BCD97] and [Tra21] for more on the eikonal and other Hamilton-Jacobi equations.

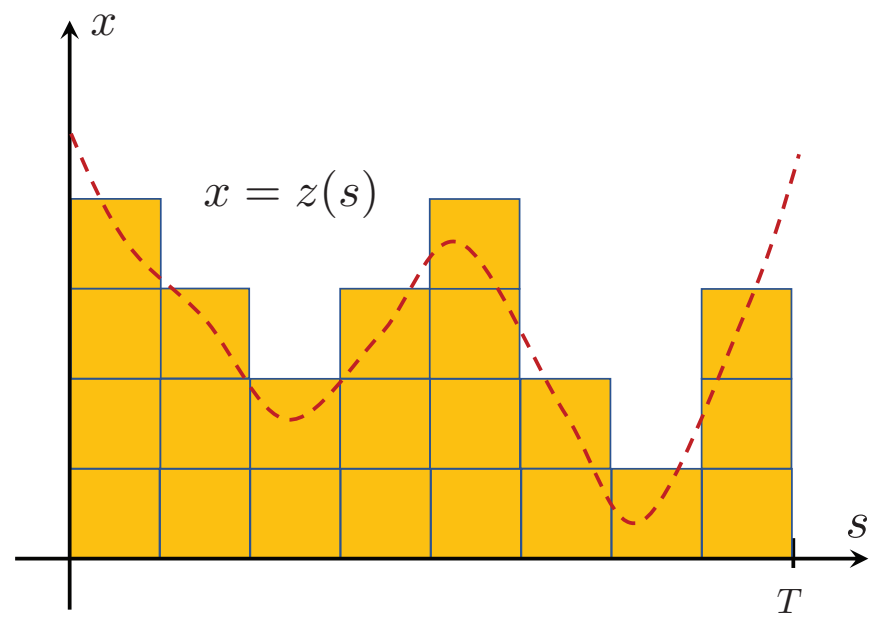

Figure 4. A graph $x=z(s)$ of the amount of a commodity produced by a hypothetical factory. The boxes represent a changing inventory over time.

2.2. Optimal production of a commodity. A basic problem in economics is to minimize the costs associated with the production of a given commodity on fixed time horizon $[0, T]$. Let us represent $z(s)$ as the amount of commodity stored in inventory at time $s$, which is driven by a variable production rate $\xi \geq 0$; for simplicity, we assume there is a constant demand rate $d$. That is,

$$
\dot{z}(s)=\xi(s)-d
$$

for $s \in(0, T)$. We seek to choose $\xi$ in order to minimize the total cost

$$
\int_{0}^{T} z(s)^{+}+\frac{1}{2} \xi(s)^{2} d s
$$

modeling the sum of specific types of holding and operational costs. Here $w^{+}:=\max \{w, 0\}$, and we'll also use $w^{-}:=\max \{-w, 0\}$ below.

Considering this optimization problem for a given level of commodity $x \in \mathbb{R}$ at time $t \in[0, T]$, we are led to the function

$$
u(x, t)=\min _{\xi \geq 0}\left\{\int_{t}^{T} z(s)^{+}+\frac{1}{2} \xi(s)^{2} d s: z(t)=x\right\} .
$$

Here the minimum is taken over paths $z:[t, T] \rightarrow \mathbb{R}$ satisfying (4) for a nonnegative production rate $\xi$. It turns out that $u$ can be interpreted as a solution of the PDE

$$
-\partial_{t} u+\frac{1}{2}\left(\partial_{x} u^{-}\right)^{2}+d \partial_{x} u-x^{+}=0 .
$$

It would be interesting to know if we can find $u$ explicitly and if it will tell us something about optimal production rates $\xi$. Finally, we note that this optimization problem is a particular case of Example 2.1 in section I.2 of [FS06].

2.3. Eradicating an infectious disease. There are many optimization problems of interest in epidemiology. We encounter one when considering the following differential equations in a compartmental model:

$$
\left\{\begin{array}{l}
\dot{S}(t)=-\beta S(t) I(t)-r(t) S(t), \\
\dot{I}(t)=\beta S(t) I(t)-\gamma I(t)
\end{array}\right.
$$

$(t>0)$. Here $S(t)$ and $I(t)$ represent the respective susceptible and infected components of a population at time $t$ which is subject to an infectious disease, $\beta$ is the transmission rate, $\gamma$ is the recovery rate, and $r(t)$ represents a vaccination rate at time $t$. As we have seen in the present COVID-19 epidemic, there may be logistical constraints in administering vaccines. As a result, we will suppose for simplicity that

$$
0 \leq r(t) \leq 1
$$

for all $t \geq 0$.

Let us fix $\mu \in(0, I(0))$. Our goal is to choose a vaccination rate $r$ so that the time

$$
\tau^{r}=\min \left\{t \geq 0: I^{r}(t)=\mu\right\}
$$

it takes for the infected population $I^{r}$ to drop down to the given threshold $\mu$ is as small as possible. Here and below, 


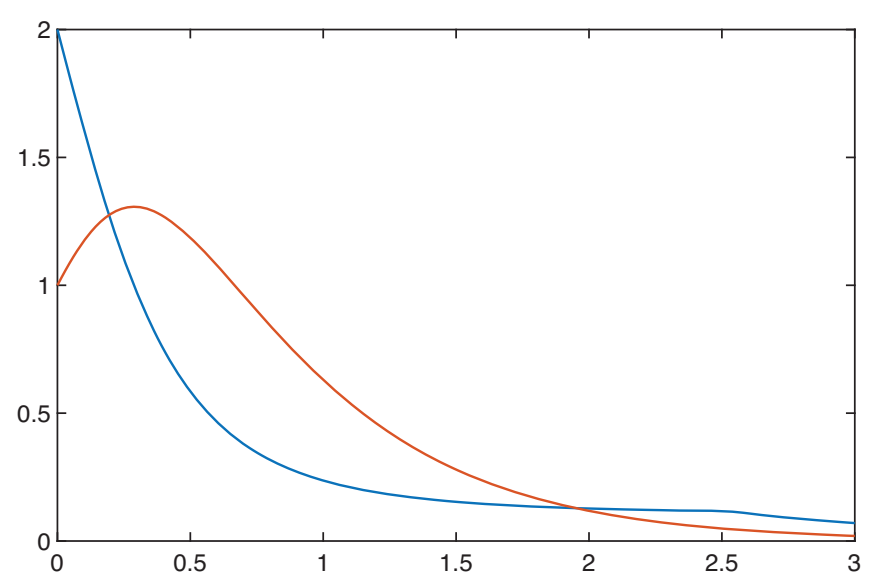

Figure 5. Solution of the differential equations (5) with $S(0)=2, I(0)=1, \beta=2$, and $\gamma=2$. The graph of $S$ is shown in blue, and the graph of $I$ is shown in red. Here the vaccination rate is $r(t)=0$ for $t \in[0,2.5]$ and $r(t)=1$ for $t \in(2.5, \infty)$.

we'll write $S^{r}$ and $I^{r}$ for the solution of (5) with vaccination rate $r$. The time $\tau^{r}$ corresponding to a minimizing $r$ is called an eradication time.

This problem was considered by a group of math biologists [BBSG17], who showed that a minimizing vaccination rate is always of the form

$$
r^{*}(t)= \begin{cases}0, & t \in[0, T], \\ 1, & t \in(T, \infty),\end{cases}
$$

for a switching time $T \geq 0$. While it seems intuitive that $T=0$ for an optimal vaccination rate, there are initial conditions $S(0), I(0)$ which correspond to positive switching times (Figure 2a of [BBSG17]). However, it is not well understood how the quadrant of initial conditions $(S(0), I(0)) \in(0, \infty) \times(\mu, \infty)$ is divided between points associated with immediate or delayed switching.

In recent work, we studied

$$
u(x, y)=\min _{0 \leq r \leq 1}\left\{\tau^{r}: S^{r}(0)=x, I^{r}(0)=y\right\}
$$

and characterized $u$ as a solution of the Hamilton-Jacobi equation

$$
\beta x y \partial_{x} u+x\left(\partial_{x} u\right)^{+}+(\gamma-\beta x) y \partial_{y} u=1
$$

for $(x, y) \in(0, \infty) \times(\mu, \infty)$ [HIP20]. It also turns out that $u$ solves another PDE which allowed us to give a new interpretation of optimal vaccination rates.

2.4. Turbulent flame fronts. In combustion science, the so-called G-equation

$$
\partial_{t} u+v \partial_{x} u+w \partial_{y} u=s \sqrt{\left(\partial_{x} u\right)^{2}+\left(\partial_{y} u\right)^{2}}
$$

is used to describe an evolving flame propagation front within a planar region [Pet00]. In particular, the level set

$$
\Gamma_{t}:=\left\{(x, y) \in \mathbb{R}^{2}: u(x, y, t)=0\right\}
$$

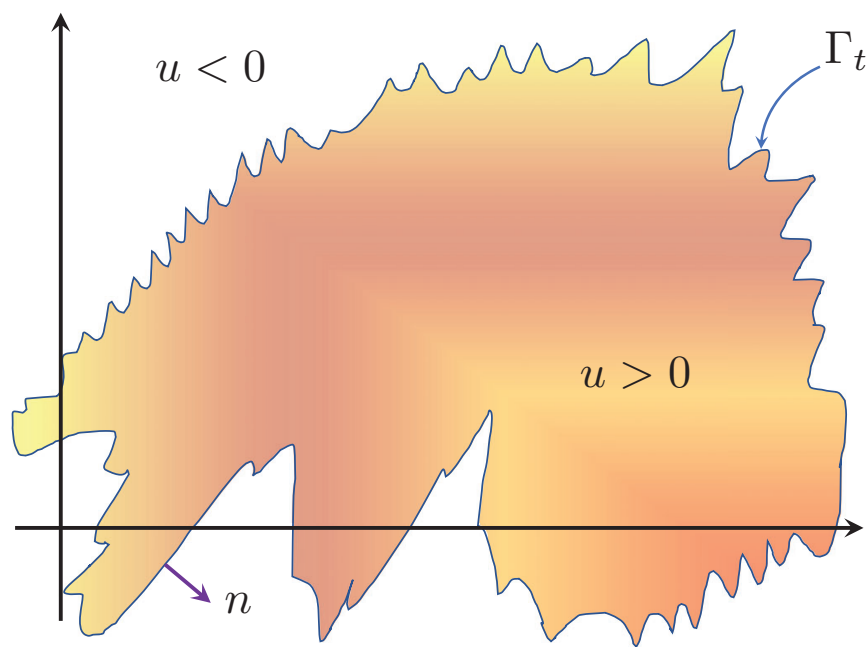

Figure 6. Schematic of the flame front $\Gamma_{t}$ determined by the zero level set $u(x, y, t)=0$; note that $n$ is the outward unit normal to $\Gamma_{t}$. This level set is time-dependent and will evolve according to the $G$-equation (6).

models the front for times $t \geq 0$. Here $v=v(x, y)$ and $w=w(x, y)$ represent the respective $x$ and $y$ coordinates of the "turbulent" velocity field $V$ of the flame; the parameter $s>0$ is the "laminar" flame speed.

The primary assumption used in the derivation of the $G$ equation is that the level set $\Gamma_{t}$ moves in direction $n$ with speed

$$
V \cdot n+s
$$

Here $n$ is the outward unit normal to $\Gamma_{t}$, which points in the negative direction of the spatial gradient of $u$ provided the burnt region corresponds to points for which $u$ is positive. A central goal of this mathematical model is to describe

$$
\lim _{t \rightarrow \infty} u(x, y, t) / t,
$$

which is interpreted as the large time flame front speed. To this end, it has been exploited that the $G$-equation is a Hamilton-Jacobi equation [NXY09].

In particular, we may represent a solution $u$ of (6) as

$$
u(x, y, t)=\max _{\alpha, \beta} u_{0}(\gamma(t), \zeta(t)) .
$$

Here $u_{0}$ is the function whose zero level set describes the front at time 0 and $\gamma, \zeta:[0, t] \rightarrow \mathbb{R}$ satisfy

$$
\left\{\begin{array}{l}
\dot{\gamma}(\tau)=-v(\gamma(\tau), \zeta(\tau))+\alpha(\tau), \\
\dot{\zeta}(\tau)=-w(\gamma(\tau), \zeta(\tau))+\beta(\tau), \\
\gamma(0)=x, \zeta(0)=y .
\end{array}\right.
$$

The "controls" $\alpha, \beta$ are required to observe the constraint

$$
\alpha(\tau)^{2}+\beta(\tau)^{2} \leq s^{2}
$$

for $\tau \in[0, t]$. Consequently, the $G$-equation is indeed a PDE which can be identified as a Hamilton-Jacobi equation. 


\section{Dynamic Programming}

Let us now return to minimizing the action associated with Newton's second law that we considered in the introduction. As before, $F=-V^{\prime}$, where $V$ is the potential energy. We will consider paths whose right endpoint is fixed and also assume that $m=1$ for convenience. As a result, our problem is to minimize

$$
\int_{0}^{t} \frac{1}{2} \dot{\zeta}(s)^{2}-V(\zeta(s)) d s
$$

among paths $\zeta:[0, t] \rightarrow \mathbb{R}$ which satisfy the constraint

$$
\zeta(t)=x
$$

for a given $x \in \mathbb{R}$ and $t \geq 0$.

To this end, we'll define the value function

$$
\begin{aligned}
u(x, t)=\min & \{g(\zeta(0)) \\
& \left.+\int_{0}^{t} \frac{1}{2} \dot{\zeta}(s)^{2}-V(\zeta(s)) d s: \zeta(t)=x\right\} .
\end{aligned}
$$

Notice that we've included a function $g: \mathbb{R} \rightarrow \mathbb{R}$ into our minimization problem. This is done with a bit of foresight, and we hope the reader will come to appreciate this addition. We also note that this definition of $u(x, t)$ should be made with an infimum rather than a minimum, since we have not verified the existence of a minimizing path. We won't lose much by this omission as it is fairly routine to find conditions on $g$ and $V$ so that the minimization problem associated with $u(x, t)$ has a solution.

3.1. Deriving the Hamilton-Jacobi equation. A key insight made by Bellman is that the values of $u(\cdot, s)$ determine $u(x, t)$ for $s<t$ [Bel54]. Specifically, there is a relationship between the prior optimal values $u(\cdot, s)$ and the current one $u(x, t)$. In general, this is called the dynamic programming principle. For our particular problem, it takes the form

$$
\begin{aligned}
u(x, t)=\min & \{u(\zeta(s), s) \\
& \left.+\int_{s}^{t} \frac{1}{2} \dot{\zeta}(\tau)^{2}-V(\zeta(\tau)) d \tau: \zeta(t)=x\right\}
\end{aligned}
$$

for $0 \leq s<t$.

Since $\zeta(t)=x$ for any admissible path in (8), we can rewrite the dynamic programming principle above as

$$
\begin{aligned}
0=\min \{ & -\frac{u(\zeta(t), t)-u(\zeta(s), s)}{t-s} \\
& \left.+\frac{1}{t-s} \int_{s}^{t} \frac{1}{2} \dot{\zeta}(\tau)^{2}-V(\zeta(\tau)) d \tau: \zeta(t)=x\right\}
\end{aligned}
$$

Assuming that $u$ is differentiable at $(x, t)$ and that $v=\dot{\zeta}(t)$ exists, we also find

$$
\begin{aligned}
\lim _{s \rightarrow t^{-}}\{- & \frac{u(\zeta(t), t)-u(\zeta(s), s)}{t-s} \\
& \left.+\frac{1}{t-s} \int_{s}^{t} \frac{1}{2} \dot{\zeta}(\tau)^{2}-V(\zeta(\tau)) d \tau\right\} \\
& =-\partial_{t} u(x, t)-v \partial_{x} u(x, t)+\frac{1}{2} v^{2}-V(x) .
\end{aligned}
$$

If we are allowed to interchange this limit and taking the minimum in (9), then

$$
\begin{aligned}
0= & \lim _{s \rightarrow t^{-}} \min \left\{-\frac{u(\zeta(t), t)-u(\zeta(s), s)}{t-s}\right. \\
& \left.+\frac{1}{t-s} \int_{s}^{t} \frac{1}{2} \dot{\zeta}(\tau)^{2}-V(\zeta(\tau)) d \tau: \zeta(t)=x\right\} \\
= & \min _{v \in \mathbb{R}}\left\{-\partial_{t} u(x, t)-v \partial_{x} u(x, t)+\frac{1}{2} v^{2}-V(x)\right\} \\
= & -\partial_{t} u(x, t)-\frac{1}{2}\left(\partial_{x} u(x, t)\right)^{2}-V(x) .
\end{aligned}
$$

As a result, dynamic programming heuristically implies the Hamilton-Jacobi equation (3).

3.2. Action-minimizing paths. It is also not hard to check that if $\zeta$ is an action-minimizing path in (7), $\left.\zeta\right|_{[s, t]}$ is also a minimizer in (8) for any $0 \leq s<t$. Making use of this fact, we can apply the technique used to derive the least action principle to (8) and show

$$
\dot{\gamma}(s)=\partial_{x} u(\gamma(s), s), \quad s \in(0, t),
$$

for any action-minimizing path $\gamma$. This conclusion assumes differentiability of the value function along minimizing trajectories. We also note that the necessary condition (10) is a part of a more general set of conditions due to Pontryagin (as detailed in Chapter 1 of [FSO6]).

In view of (7), we see that $u(x, 0)=g(x)$. Therefore, $u$ is a candidate for a solution of the initial value problem

$$
\left\{\begin{array}{l}
\partial_{t} u+\frac{1}{2}\left(\partial_{x} u\right)^{2}+V(x)=0 \quad \text { in } \mathbb{R} \times(0, \infty) \\
u(x, 0)=g(x)
\end{array}\right.
$$

Alternatively, if $w$ is a continuously differentiable solution of this initial value problem and $\zeta:[0, t] \rightarrow \mathbb{R}$ is a path 
with $\zeta(t)=x$, then

$$
\begin{aligned}
w(x, t) & w(\zeta(t), t) \\
= & w(\zeta(0), 0)+\int_{0}^{t} \frac{d}{d s} w(\zeta(s), s) d s \\
= & g(\zeta(0)) \\
& +\int_{0}^{t}\left(\partial_{t} w(\zeta(s), s)+\partial_{x} w(\zeta(s), s) \dot{\zeta}(s)\right) d s \\
\leq & g(\zeta(0)) \\
& +\int_{0}^{t}\left(\partial_{t} w(\zeta(s), s)+\frac{1}{2} \partial_{x} w(\zeta(s), s)^{2}+\frac{1}{2} \dot{\zeta}(s)^{2}\right) d s \\
= & g(\zeta(0))+\int_{0}^{t}\left(\frac{1}{2} \dot{\zeta}(s)^{2}-V(\zeta(s))\right) d s .
\end{aligned}
$$

Here we used the elementary inequality $a b \leq \frac{1}{2}\left(a^{2}+b^{2}\right)$.

As $\zeta$ was arbitrary,

$$
w(x, t) \leq u(x, t)
$$

The computation above also shows that if an admissible path $\gamma$ satisfies $\dot{\gamma}(s)=\partial_{x} w(\gamma(s), s)$ for $s \in(0, t)$, then $w(x, t)=u(x, t)$. It follows that (10) is a necessary and sufficient condition for optimality when $u$ is a continuously differentiable solution of the initial value problem (11). In particular, if we know the value function, we can attempt to design an action-minimizing path in (7) by solving (10) subject to the endpoint constraint $\gamma(t)=x$.

3.3. Viscosity solutions. A question we have not addressed is: does $u$ actually solve the initial value problem (11)? It turns out that the value function may not be everywhere differentiable even when $g$ and $V$ are continuously differentiable. For example, if $V \equiv 0$ and

$$
g(x)= \begin{cases}-\frac{1}{2} x^{2}, & |x|<1, \\ \frac{1}{2}-|x|, & |x| \geq 1,\end{cases}
$$

then the corresponding value function

$$
u(x, t)= \begin{cases}-\frac{1}{2(1-t)} x^{2}, & |x|<1-t, \\ \frac{1-t}{2}-|x|, & |x| \geq 1-t,\end{cases}
$$

is not differentiable at any point of the form $(0, t)$ for $t \geq 1$. Therefore, we cannot completely rely on our previous computations. However, it was noted by Crandall and Lions [CL83] that

$$
\partial_{t} \varphi\left(x_{0}, t_{0}\right)+\frac{1}{2}\left(\partial_{x} \varphi\left(x_{0}, t_{0}\right)\right)^{2}+V\left(x_{0}\right) \leq 0,
$$

whenever $\varphi: \mathbb{R} \times(0, \infty) \rightarrow \mathbb{R}$ is smooth and $u-\varphi$ has a local maximum at $\left(x_{0}, t_{0}\right)$ in $\mathbb{R} \times(0, \infty)$ and that

$$
\partial_{t} \varphi\left(x_{0}, t_{0}\right)+\frac{1}{2}\left(\partial_{x} \varphi\left(x_{0}, t_{0}\right)\right)^{2}+V\left(x_{0}\right) \geq 0,
$$

if instead $u-\varphi$ has a local minimum at $\left(x_{0}, t_{0}\right)$.

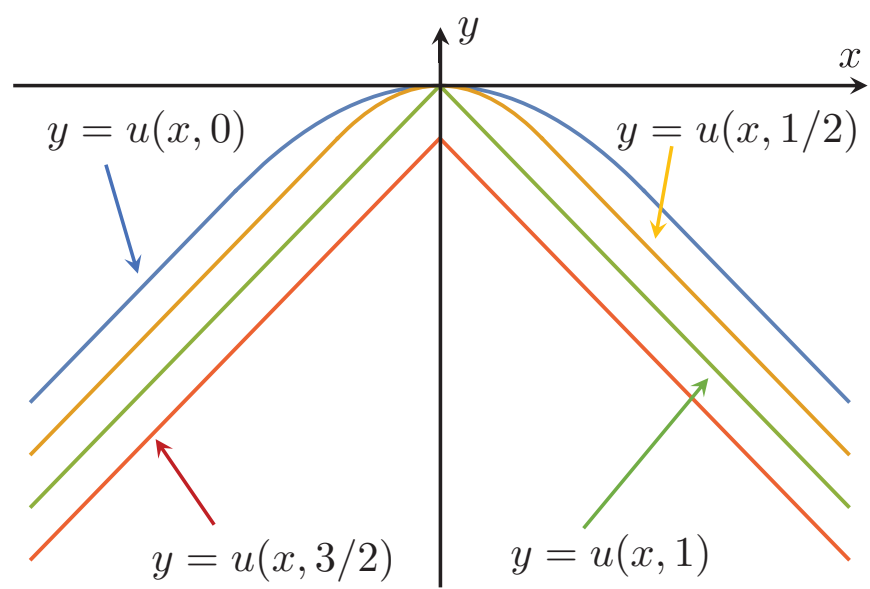

Figure 7. Profiles $y=u(x, t)$ of the value function corresponding to $V \equiv 0$ and $g$ defined in (12) for $t=0,1 / 2,1$, and $3 / 2$. For this example, the graph $y=u(x, t)$ is continuously differentiable for each $0 \leq t<1$ but not differentiable at $x=0$ for $t \geq 1$. This example illustrates that the value function may not be differentiable even if $g$ and $V$ are.

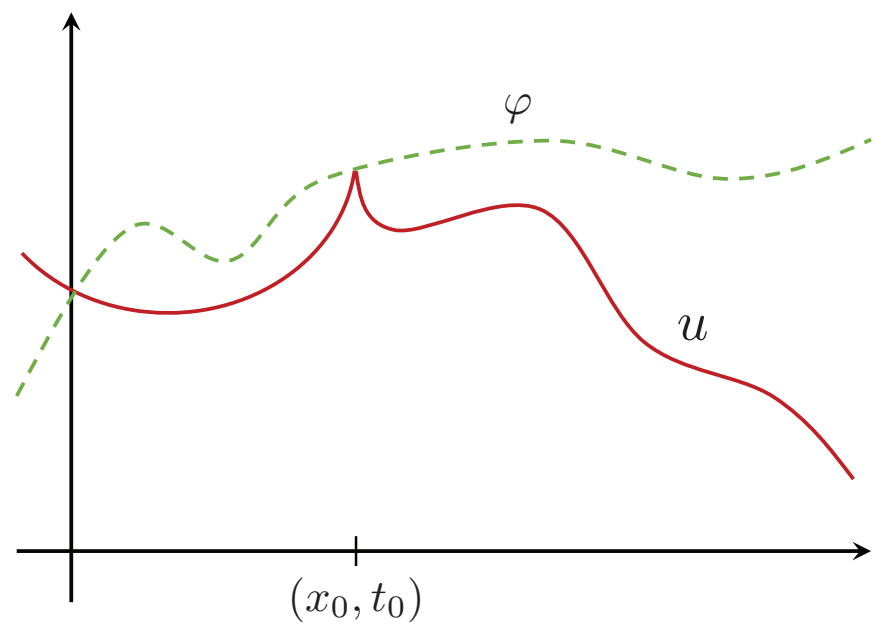

Figure 8. The value function $u$ being touched by a smooth function $\varphi$ from above at a point $\left(x_{0}, t_{0}\right)$. Note in particular, that $u$ may not be differentiable at $\left(x_{0}, t_{0}\right)$. Nevertheless, the inequality $\partial_{t} \varphi\left(x_{0}, t_{0}\right)+\frac{1}{2}\left(\partial_{x} \varphi\left(x_{0}, t_{0}\right)\right)^{2}+V\left(x_{0}\right) \leq 0$ holds.

That is to say, if $u$ can be touched from above or below by a smooth function $\varphi$ at the point $\left(x_{0}, t_{0}\right)$, then an inequality related to the Hamilton-Jacobi equation must hold. It is also the case that if $u$ happens to be differentiable at $\left(x_{0}, t_{0}\right)$, then it satisfies the Hamilton-Jacobi equation at $\left(x_{0}, t_{0}\right)$. Consequently, this notion of solution extends the "classical" notion of what it means for a function to be a solution. Any function satisfying both of these touching conditions is called a viscosity solution for reasons we'll get to shortly.

We also mention that viscosity solutions as defined by Crandall and Lions was preceded by virtually the same notion Evans used to show existence of solutions to various 


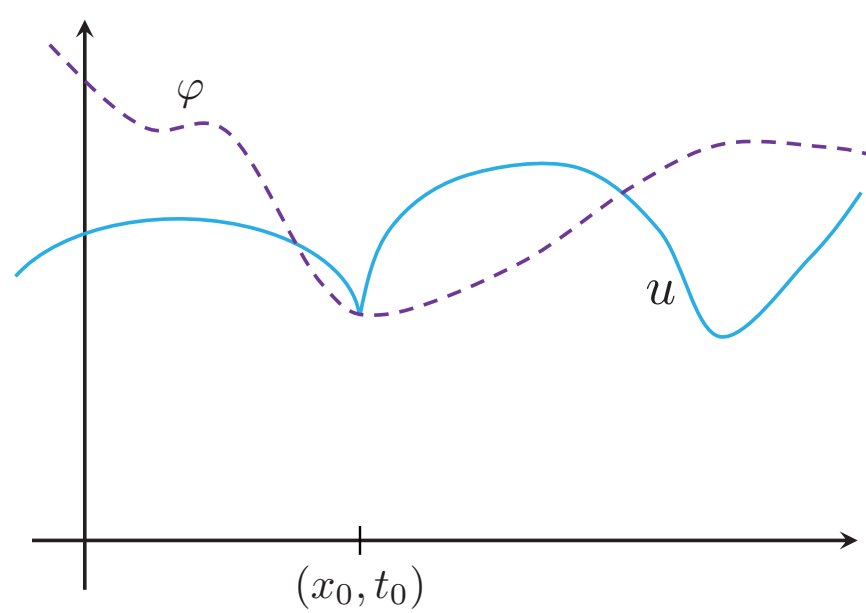

Figure 9. The value function $u$ being touched by a smooth function $\varphi$ from below at a point $\left(x_{0}, t_{0}\right)$. As $u$ is a viscosity solution of the Hamilton-Jacobi equation (3) (with $m=1$ ), the inequality $\partial_{t} \varphi\left(x_{0}, t_{0}\right)+\frac{1}{2}\left(\partial_{x} \varphi\left(x_{0}, t_{0}\right)\right)^{2}+V\left(x_{0}\right) \geq 0$ must hold.

equations including Hamilton-Jacobi equations [Eva80]. The major contribution of Crandall and Lions was that they verified this notion yields uniqueness of solutions which are given by the very formula used to derive the Hamilton-Jacobi equation. Moreover, viscosity solutions have been used to properly interpret solutions of many other nonlinear PDEs which admit some sort of "comparison principle" [CIL92].

Both Crandall and Lions [CL83] and Evans [Eva80] cite the vanishing viscosity method as the reason why they arrived at viscosity solutions. This amounts to considering the initial value problem

$$
\left\{\begin{array}{l}
\partial_{t} u^{\epsilon}+\frac{1}{2}\left(\partial_{x} u^{\epsilon}\right)^{2}+V(x)=\epsilon \partial_{x}^{2} u^{\epsilon} \quad \text { in } \mathbb{R} \times(0, \infty), \\
u^{\epsilon}(x, 0)=g(x)
\end{array}\right.
$$

for an "artificial viscosity" $\epsilon>0$. The expectation is that $u^{\epsilon}$ is smooth in $\mathbb{R} \times(0, \infty)$ and that

$$
u^{\epsilon} \rightarrow u
$$

uniformly on bounded subsets of $\mathbb{R} \times[0, \infty)$. In this case, if $u-\varphi$ has a strict local maximum at $\left(x_{0}, t_{0}\right) \in \mathbb{R} \times(0, \infty)$, then a simple analysis can be used to show that there is a sequence of $\epsilon$ tending to 0 and local maximization points $\left(x_{\epsilon}, t_{\epsilon}\right)$ for $u^{\epsilon}-\varphi$ such that $\left(x_{\epsilon}, t_{\epsilon}\right) \rightarrow\left(x_{0}, t_{0}\right)$. Consequently,

$$
\left\{\begin{array}{l}
\partial_{t} u^{\epsilon}\left(x_{\epsilon}, t_{\epsilon}\right)=\partial_{t} \varphi\left(x_{\epsilon}, t_{\epsilon}\right), \\
\partial_{x} u^{\epsilon}\left(x_{\epsilon}, t_{\epsilon}\right)=\partial_{x} \varphi\left(x_{\epsilon}, t_{\epsilon}\right), \\
\partial_{x}^{2} u^{\epsilon}\left(x_{\epsilon}, t_{\epsilon}\right) \leq \partial_{x}^{2} \varphi\left(x_{\epsilon}, t_{\epsilon}\right),
\end{array}\right.
$$

and in view of (14),

$$
\partial_{t} \varphi\left(x_{\epsilon}, t_{\epsilon}\right)+\frac{1}{2}\left(\partial_{x} \varphi\left(x_{\epsilon}, t_{\epsilon}\right)\right)^{2}+V\left(x_{\epsilon}\right) \leq \epsilon \partial_{x}^{2} \varphi\left(x_{\epsilon}, t_{\epsilon}\right) .
$$

Sending $\epsilon \rightarrow 0$ leads to (13).

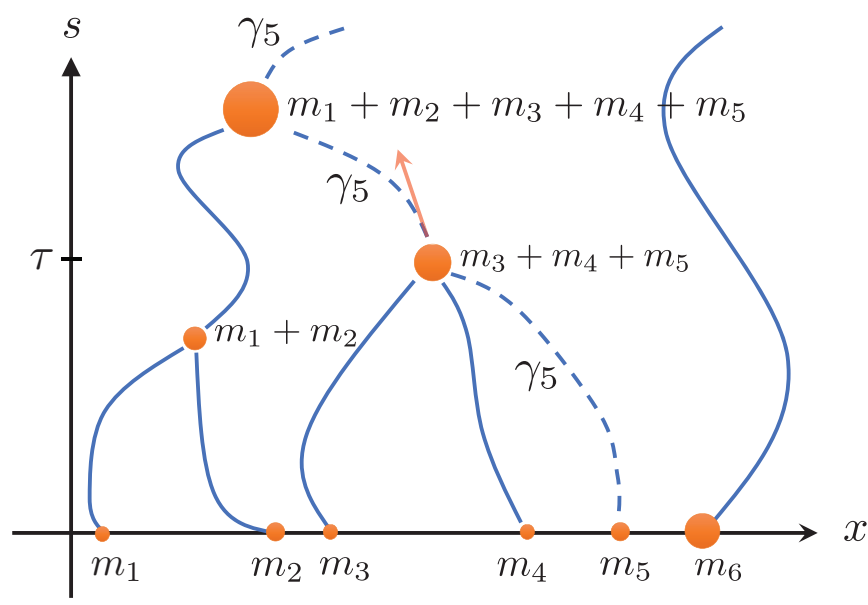

Figure 10. Sticky particle trajectories $\gamma_{1}, \ldots, \gamma_{6}$ determined by (15) when there are no collisions and by the physics of perfectly inelastic collisions otherwise. Right after the collision between particles 3,4 , and 5 at time $\tau$, the slope of $\gamma_{5}$ satisfies $\left(m_{3}+m_{4}+m_{5}\right) \dot{\gamma}_{5}(\tau+)=m_{3} \dot{\gamma}_{3}(\tau-)+m_{4} \dot{\gamma}_{4}(\tau-)+m_{5} \dot{\gamma}_{5}(\tau-)$.

\section{Interacting Particles}

Hamilton-Jacobi equations also arise in the study of interacting particles. We will explore one such connection by considering $N$ particles labeled $1, \ldots, N$ with respective masses $m_{1}, \ldots, m_{N}$ which move along the real line. We will assume that these point masses interact pairwise and undergo perfectly inelastic collisions when they collide. As the particles will stick together upon collision, we will call them a collection of sticky particles.

Let us denote $\gamma_{1}, \ldots, \gamma_{N}$ as the coordinates of the respective particles and suppose that the pairwise interaction is governed by a symmetric potential function $\Phi$. Specifically, we will presume that the force exerted on particle $i$ by particle $j$ is equal to

$$
-m_{i} m_{j} \Phi^{\prime}\left(\gamma_{i}-\gamma_{j}\right)
$$

As a result, the trajectories satisfy the Newton system

$$
m_{i} \ddot{\gamma}_{i}(s)=-\sum_{j \neq i} m_{i} m_{j} \Phi^{\prime}\left(\gamma_{i}(s)-\gamma_{j}(s)\right)
$$

$(i=1, \ldots, N)$ for times $s$ in between collisions.

Upon collision, particles stick together, so their corresponding paths coincide thereafter. The slopes of these trajectories are also determined by the physics of perfectly inelastic collisions. For example, suppose that the particles with respective masses $m_{1}, \ldots, m_{k}$ collide at time $\tau$. The resulting particle has mass $m_{1}+\cdots+m_{k}$, and its instantaneous velocity $v$ satisfies

$$
m_{1} \dot{\gamma}_{1}(\tau-)+\cdots+m_{k} \dot{\gamma}_{k}(\tau-)=\left(m_{1}+\cdots+m_{k}\right) v .
$$

That is, $v$ is the mass average of the colliding particles' velocities just prior to the collision. Observe that mass and momentum are conserved through the collision. 
4.1. Pressureless-Euler equations. The sticky particle trajectories described above can be used to design a solution of the pressureless-Euler equations

$$
\left\{\begin{array}{c}
\partial_{S} \rho+\partial_{x}(\rho v)=0 \\
\partial_{s}(\rho v)+\partial_{x}\left(\rho v^{2}\right)=-\rho\left(\Phi^{\prime} * \rho\right)
\end{array}\right.
$$

in $\mathbb{R} \times(0, \infty)$ [Hyn20, BGSW13]. Here $\rho$ represents the mass density of the particles and $v$ is the corresponding local velocity function. In general, this PDE system describes an evolving mass distribution in which particles interact pairwise according to potential $\Phi$ and undergo perfectly inelastic collisions when they collide. It reduces to sticky particle dynamics in the special case of finitely many evolving point masses.

As the pressureless-Euler equations (16) are related to the Newton system (15), it is natural to seek a least action principle as discussed in the introduction. To this end, we will restrict attention to solutions which have finite mass. Since we expect that the total mass is conserved along the flow, we may normalize it to unity. This basic observation leads us to consider the space $\mathcal{P}_{2}(\mathbb{R})$ of Borel probability measures $\mu$ on $\mathbb{R}$ with finite second moment

$$
\int_{\mathbb{R}} x^{2} d \mu(x)<\infty .
$$

We recall that $\mathcal{P}_{2}(\mathbb{R})$ is a complete metric space when endowed with the Wasserstein distance

$$
W_{2}(\mu, v)=\min _{\pi}\left(\iint_{\mathbb{R}^{2}}(x-y)^{2} d \pi(x, y)\right)^{1 / 2} .
$$

Here the minimum is taken over Borel probability measures $\pi$ on $\mathbb{R}^{2}$ whose first and second marginals are $\mu$ and $\nu$, respectively. At each $\mu, \mathcal{P}_{2}(\mathbb{R})$ also has a tangent space $\operatorname{Tan}_{\mu} \mathcal{P}_{2}(\mathbb{R})$ consisting of all $v \in L^{2}(\mu)$ such that

$$
\int_{\mathbb{R}} v(x)^{2} d \mu(x) \leq \int_{\mathbb{R}} w(x)^{2} d \mu(x)
$$

whenever $w \in L^{2}(\mu)$ and

$$
\int_{\mathbb{R}} \phi^{\prime}(x) v(x) d \mu(x)=\int_{\mathbb{R}} \phi^{\prime}(x) w(x) d \mu(x)
$$

for each smooth $\phi: \mathbb{R} \rightarrow \mathbb{R}$ which vanishes outside of a bounded interval. We will see below why it makes sense to identify this tangent space.

The action integral associated with the pressurelessEuler equations is

$$
\begin{aligned}
\mathcal{A}_{t}(\sigma):=\int_{0}^{t} & \left\{\int_{\mathbb{R}} \frac{1}{2} w(x, s)^{2} d \sigma_{s}(x)\right. \\
& \left.-\frac{1}{2} \iint_{\mathbb{R}^{2}} \Phi(x-y) d \sigma_{s}(x) d \sigma_{s}(y)\right\} d s .
\end{aligned}
$$

Here $\sigma:[0, t] \rightarrow \mathcal{P}_{2}(\mathbb{R}) ; s \mapsto \sigma_{s}$ is a path and $w:$ $\mathbb{R} \times[0, t] \rightarrow \mathbb{R}$ is a Borel function such that the continuity equation

$$
\partial_{s} \sigma+\partial_{x}(\sigma w)=0
$$

holds on $\mathbb{R} \times(0, t)$. For a given $\sigma$, there can be many $w$ such that the above continuity equation holds. Among these, there is a unique "tangent" velocity function $w$ with

$$
w(\cdot, s) \in \operatorname{Tan}_{\sigma_{s}} \mathcal{P}_{2}(\mathbb{R})
$$

and that has the smallest kinetic energy

$$
\frac{1}{2} \int_{\mathbb{R}} w(x, s)^{2} d \sigma_{s}(x)
$$

for almost every $s \in[0, t]$ (section 8.3 of [AGS05]). As a result, we always will associate this $w$ to $\sigma$ in the definition of $\mathcal{A}_{t}(\sigma)$.

It can be shown that if $\rho:[0, t] \rightarrow \mathcal{P}_{2}(\mathbb{R})$ is a path such that

$$
\mathcal{A}_{t}(\rho) \leq \mathcal{A}_{t}(\sigma)
$$

for all paths $\sigma:[0, t] \rightarrow \mathcal{P}_{2}(\mathbb{R})$ with $\sigma_{t}=\mu$, then $\rho$ and its tangent velocity function $v$ form a solution pair of the pressureless-Euler equations in $\mathbb{R} \times(0, t)$ (Theorem 3.9 of [GNT08]). That is, a least action principle for the pressureless-Euler equations does indeed hold.

4.2. Hamilton-Jacobi equation in the space of probability measures. It is also natural to study the associated value function

$$
\mathcal{U}(\mu, t):=\min \left\{\mathcal{G}\left(\sigma_{0}\right)+\mathcal{A}_{t}(\sigma): \sigma_{t}=\mu\right\}
$$

for a given $\mu \in \mathcal{P}_{2}(\mathbb{R})$, time $t \geq 0$, and function $\mathcal{G}$ : $\mathcal{P}_{2}(\mathbb{R}) \rightarrow \mathbb{R}$. Under reasonable assumptions on $\mathcal{G}$ and $\Phi$, $\mathcal{U}$ satisfies a dynamic programming principle and is a type of viscosity solution of the abstract Hamilton-Jacobi equation

$$
\begin{aligned}
0 & =\partial_{t} \mathcal{U}(\mu, t)+\frac{1}{2} \int_{\mathbb{R}} \partial_{\mu} u(\mu, t)^{2} d \mu \\
& +\frac{1}{2} \iint_{\mathbb{R}^{2}} \Phi(x-y) d \mu(x) d \mu(y)
\end{aligned}
$$

for $(\mu, t) \in \mathcal{P}_{2}(\mathbb{R}) \times(0, \infty)$ [GNT08, HK15]. Moreover, $u$ satisfies the initial condition

$$
\mathcal{U}(\mu, 0)=\mathcal{G}(\mu) .
$$

We say that $\mathcal{V}$ is differentiable at $\mu$ if there is $\xi \in$ $\operatorname{Tan}_{\mu} \mathcal{P}_{2}(\mathbb{R})$ with the property that for each $\epsilon$ there is $\delta$ such that if $W_{2}(\mu, \nu)<\delta$, then

$$
\frac{\left|\mathcal{V}(\nu)-\mathcal{V}(\mu)-\iint_{\mathbb{R}^{2}} \xi(x)(y-x) d \pi(x, y)\right|}{W_{2}(\mu, \nu)}<\epsilon
$$

for all optimal $\pi$ for $W_{2}(\mu, \nu)$. In this case, it is not hard to check that

$$
\xi=\partial_{\mu} V(\mu)
$$


is uniquely determined as an $L^{2}(\mu)$ function. The value function $U$ is a type of viscosity solution of (17) which is based on this notion of differentiability. And if $\mathcal{U}$ happens to be differentiable, we can attempt to build an actionminimizing trajectory $\rho$ for $\mathcal{U}(\mu, t)$ by solving

$$
\partial_{s} \rho+\partial_{x}(\rho v)=0
$$

on $\mathbb{R} \times(0, t)$ subject to the endpoint constraint $\rho_{t}=\mu$ with

$$
v(\cdot, s)=\partial_{\mu} \mathcal{U}\left(\rho_{s}, s\right)
$$

for $0 \leq s \leq t$. This would be the analog of condition (10).

Understanding solutions of the Hamilton-Jacobi equation (17) and its variants from an intrinsic point of view is currently an active area of research with much room for growth.

\section{Game Theory}

PDEs also play an important role in the theory of differential games [Isa65]. In this section, we will focus on noncooperative, differential games as they involve an interesting system of Hamilton-Jacobi equations. To this end, we consider $N$ players labeled $i=1, \ldots, N$, and for the moment, we fix a time $t \in[0, T]$ and an $N$-tuple $x=\left(x_{1}, \ldots, x_{N}\right) \in \mathbb{R}^{N}$.

We will assume that player $i$ has the cost functional

$$
g_{t}^{i}(\zeta):=\int_{t}^{T} \frac{1}{2} \dot{\zeta}_{i}(s)^{2} d s+g_{i}(\zeta(T))
$$

for $i=1, \ldots, N$. Here $\frac{1}{2} \dot{\zeta}_{i}(s)^{2}$ is player $i$ 's running cost per unit time and $g_{i}: \mathbb{R}^{N} \rightarrow \mathbb{R}$ is player $i^{\prime}$ 's terminal cost function. This player's goal is to select a strategy $\zeta_{i}:[t, T] \rightarrow \mathbb{R}$ with $\zeta_{i}(t)=x_{i}$ to make her cost as small as possible. We have also written

$$
\zeta:=\left(\zeta_{1}, \ldots, \zeta_{N}\right)
$$

as the $N$-tuple of strategies of all $N$ players.

Notice that the $i$ th player's cost functional depends on her own strategy $\zeta_{i}$ and the strategies

$$
\zeta_{1}, \ldots, \zeta_{i-1}, \zeta_{i+1}, \ldots, \zeta_{N}
$$

of the other players. As in the theory pioneered by Nash, we can try to find an $N$-tuple of paths $\gamma=\left(\gamma_{1}, \ldots, \gamma_{N}\right)$ such that

$$
\partial_{t}^{i}(\gamma) \leq \partial_{t}^{i}\left(\zeta_{i} ; \gamma_{-i}\right)
$$

for $i=1, \ldots, N$. Here

$$
\left(\zeta_{i} ; \gamma_{-i}\right):=\left(\gamma_{1}, \ldots, \gamma_{i-1}, \zeta_{i}, \gamma_{i+1}, \ldots, \gamma_{N}\right)
$$

Such an $N$-tuple is called a Nash equilibrium. Note in particular, that if the $i$ th player unilaterally changes her strategy from $\gamma_{i}$, her cost can only increase.

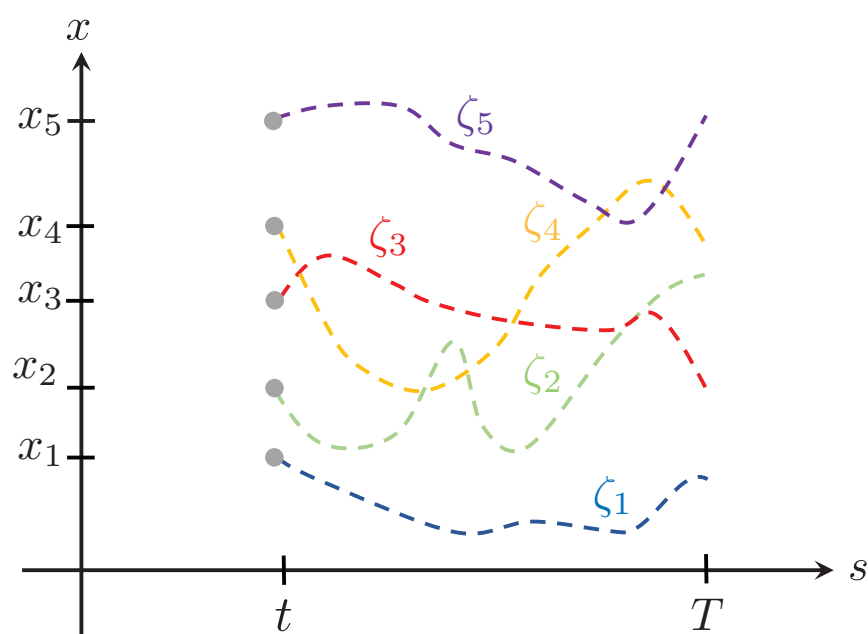

Figure 11. A collection of strategies $\zeta=\left(\zeta_{1}, \ldots, \zeta_{5}\right)$ for a 5-player, noncooperative differential game on the horizon $[t, T]$. The $i$ th player assumes a strategy $\zeta_{i}:[t, T] \rightarrow \mathbb{R}$ with $\zeta_{i}(t)=x_{i}$ in an attempt to reduce her individual cost $\mathcal{\partial}_{i}(\zeta)$.

5.1. Nash system. It turns out that a closely related equilibrium concept is associated with a system of PDEs much in the same way that minimizing the action $\mathcal{S}_{t}(2)$ is connected to the Hamilton-Jacobi equation (3). The Nash system is given by

$$
-\partial_{t} u_{i}+\frac{1}{2}\left(\partial_{x_{i}} u_{i}\right)^{2}+\sum_{j \neq i} \partial_{x_{j}} u_{j} \partial_{x_{j}} u_{i}=0
$$

for $i=1, \ldots, N$, and it holds in $\mathbb{R}^{N} \times(0, T)$. The unknowns are $N$ functions

$$
u_{1}, \ldots, u_{N}: \mathbb{R}^{N} \times(0, T] \rightarrow \mathbb{R} .
$$

It is of interest to deduce whether or not a solution exists for given terminal conditions. Of course, a resolution will depend on what type of solution is being discussed.

Let us again fix a time $t \in[0, T]$ and $x \in \mathbb{R}^{N}$ and for the moment suppose that there is a continuously differentiable solution set $u_{1}, \ldots, u_{N}$ which satisfies

$$
u_{i}(y, T)=g_{i}(y)
$$

for $i=1, \ldots, N$ and $y \in \mathbb{R}^{N}$. If in addition we can solve the differential equations

$$
\left\{\begin{array}{l}
\dot{\gamma}_{i}(s)=-\partial_{x_{i}} u_{i}(\gamma(s), s), s \in(t, T) \\
\gamma_{i}(t)=x_{i}
\end{array}\right.
$$

for $i=1, \ldots, N$, then $\gamma=\left(\gamma_{1}, \ldots, \gamma_{N}\right)$ is what is known as a feedback Nash equilibrium. That is, it satisfies

$$
\partial_{t}^{i}(\gamma) \leq \partial_{t}^{i}\left(\zeta_{i} ; \hat{\gamma}_{-i}\right)
$$

for $i=1, \ldots, N$ and any $N$-tuple $\zeta=\left(\zeta_{1}, \ldots, \zeta_{N}\right)$ with $\zeta(t)=$ $x$. In this case,

$$
u_{i}(x, t)=g_{t}^{i}(\gamma)
$$

for $i=1, \ldots, N$. 
Of course, we need to specify $\hat{\gamma}_{-i}$ in (19). For a given $i \in\{1, \ldots, N\}$ and $\zeta_{i}:[t, T] \rightarrow \mathbb{R}$,

$$
\hat{\gamma}_{-i}:[t, T] \rightarrow \mathbb{R}^{N-1}
$$

is a path such that

$$
\left\{\begin{array}{l}
\dot{\hat{\gamma}}_{j}(s)=-\partial_{x_{j}} u_{j}\left(\zeta_{i}(s) ; \hat{\gamma}_{-i}(s), s\right), s \in(t, T), \\
\hat{\gamma}_{j}(t)=x_{j}
\end{array}\right.
$$

for all $j \neq i$. Consequently, we can interpret a feedback Nash equilibrium $\gamma$ as an $N$-tuple of strategies with the property that if the $i$ th coordinate function of the map

$$
(x, t) \mapsto\left(-\partial_{x_{1}} u_{1}(x, t), \ldots,-\partial_{x_{N}} u_{N}(x, t)\right)
$$

which drives the system of differential equations (18) is changed, the cost of the $i$ th player cannot decrease.

We acknowledge that this discussion is purely heuristic. The existence of solutions to the Nash system for given terminal values has yet to be established; unfortunately, we do not know of an analog of viscosity solutions for this system of equations. In particular, there is no known way to interpret the cost functionals $\partial_{t}^{1}, \ldots, \partial_{t}^{N}$, evaluated along appropriate feedback Nash equilibria, as a solution of the Nash system.

5.2. The master equation. Something interesting occurs if our noncooperative differential game has many players and a certain symmetry. For illustrative purposes, we'll assume there is $\mathcal{G}: \mathbb{R} \times \mathcal{P}_{2}(\mathbb{R}) \rightarrow \mathbb{R}$ such that

$$
g_{i}(x)=\mathcal{G}\left(x_{i}, \rho_{N}^{i}\right)
$$

for $i=1, \ldots, N$. We have written

$$
\rho_{N}^{i}=\frac{1}{N-1} \sum_{j \neq i} \delta_{x_{j}}
$$

for the empirical distribution of all players except player $i$. As $N \rightarrow \infty$, Lions established the approximation

$$
u_{i}(x, t) \approx \mathcal{u}\left(x_{i}, \rho_{N}^{i}, t\right)
$$

when various assumptions are made (section 7 of [Car12]). Here $\mathcal{U}$ solves the master equation

$$
\begin{aligned}
0= & -\partial_{t} u(x, \mu, t)+\frac{1}{2}\left(\partial_{x} u(x, \mu, t)\right)^{2} \\
& +\int_{\mathbb{R}} \partial_{x} u(y, \mu, t)\left[\partial_{\mu} u(x, \mu, t)\right](y) d \mu(y)
\end{aligned}
$$

in $\mathbb{R} \times \mathcal{P}_{2}(\mathbb{R}) \times(0, T)$ and satisfies the terminal condition

$$
\mathcal{U}(x, \mu, T)=\mathcal{G}(x, \mu) .
$$

This formal computation has been made rigorous for related models in a multidimensional setting [CDLL19] (with appropriate second derivative terms and with a compact underlying space). The theory for these limits is called mean field games [LL07].
The connection between the master equation and game theory is as follows. Fix $x \in \mathbb{R}, t \geq 0$, and $\mu \in \mathcal{P}_{2}(\mathbb{R})$ and set

$$
\mathcal{J}_{t}(\xi, \sigma):=\int_{t}^{T} \frac{1}{2} \dot{\xi}(s)^{2} d s+\mathcal{G}\left(\xi(T), \sigma_{T}\right) .
$$

Here $\xi:[t, T] \rightarrow \mathbb{R}$ is a path with $\xi(t)=x$, and $\sigma:$ $[t, T] \rightarrow \mathcal{P}_{2}(\mathbb{R})$ is a path with $\sigma_{t}=\mu$. The functional $\mathcal{J}_{t}$ represents the cost of a single, representative player assuming a strategy $\xi$ on the interval $[t, T]$ while observing the distribution $\sigma$ of all other players.

Let us suppose that $U$ is a differentiable solution of the master equation (20) which satisfies the terminal condition $(21)$. If $(\gamma, \rho)$ is a pair such that

$$
\left\{\begin{array}{l}
\partial_{s} \rho-\partial_{x}\left(\rho \partial_{x} \mathcal{U}(\cdot, \rho, s)\right)=0 \quad \text { in } \mathbb{R} \times(t, T), \\
\dot{\gamma}(s)=-\partial_{x} \mathcal{U}\left(\gamma(s), \rho_{s}, s\right), \quad s \in(t, T), \\
\gamma(t)=x, \quad \rho_{t}=\mu,
\end{array}\right.
$$

then

$$
\mathcal{J}_{t}(\gamma, \rho) \leq \mathcal{J}_{t}(\xi, \rho)
$$

for any path $\xi:[t, T] \rightarrow \mathbb{R}$ with $\xi(t)=x$. Moreover,

$$
\mathcal{U}(x, \mu, t)=\mathcal{J}_{t}(\gamma, \rho) .
$$

We call such a pair $(\gamma, \rho)$ a mean field game equilibrium. In particular, solutions of the master equation give us an approach to designing these equilibria.

Mean field games is presently a topic of great interest. We also emphasize that we have only discussed one type of mean field game problem. Optimistically, we anticipate that there is still a lot to be done in terms of understanding master equations in the various game-theoretic settings in which they arise.

\section{A Closing Remark}

What we've discussed about Hamilton-Jacobi equations is just a glimpse. We didn't discuss Hamilton-JacobiBellman equations in stochastic optimal control, the theory of scalar conservation laws, homogenization, weak KAM theory, nonconvex Hamilton-Jacobi equations, numerical methods, nor applications to machine learning. Nevertheless, our wish is that this note illustrates some of the breadth and beauty of this subject. In particular, we hope that the ideas we have discussed continue to develop and bring new understanding to the research frontiers we mentioned and the many others involving HamiltonJacobi equations.

ACKNOWLEDGMENT. The author was partially supported by NSF grant DMS- 1554130 . 


\section{References}

[AGS05] Luigi Ambrosio, Nicola Gigli, and Giuseppe Savaré, Gradient flows in metric spaces and in the space of probability measures, Lectures in Mathematics ETH Zürich, Birkhäuser Verlag, Basel, 2005. MR2129498

[BCD97] Martino Bardi and Italo Capuzzo-Dolcetta, Optimal control and viscosity solutions of Hamilton-Jacobi-Bellman equations, Systems \& Control: Foundations \& Applications, Birkhäuser Boston, Inc., Boston, MA, 1997. With appendices by Maurizio Falcone and Pierpaolo Soravia, DOI 10.1007/978-0-8176-4755-1. MR1484411

[Bel54] Richard Bellman, The theory of dynamic programming, Bull. Amer. Math. Soc. 60 (1954), 503-515, DOI 10.1090/S0002-9904-1954-09848-8. MR67459

[BBSG17] Luca Bolzoni, Elena Bonacini, Cinzia Soresina, and Maria Groppi, Time-optimal control strategies in SIR epidemic models, Math. Biosci. 292 (2017), 86-96, DOI 10.1016/j.mbs.2017.07.011. MR3688684

[BGSW13] Y. Brenier, W. Gangbo, G. Savaré, and M. Westdickenberg, Sticky particle dynamics with interactions (English, with English and French summaries), J. Math. Pures Appl. (9) 99 (2013), no. 5, 577-617, DOI 10.1016/j.matpur.2012.09.013. MR3039208

[Car12] Pierre Cardaliaguet, Notes on mean field games, 2012.

[CDLL19] Pierre Cardaliaguet, François Delarue, JeanMichel Lasry, and Pierre-Louis Lions, The master equation and the convergence problem in mean field games, Annals of Mathematics Studies, vol. 201, Princeton University Press, Princeton, NJ, 2019, DOI 10.2307/j.ctvckq7qf. MR3967062

[CIL92] Michael G. Crandall, Hitoshi Ishii, and Pierre-Louis Lions, User's guide to viscosity solutions of second order partial differential equations, Bull. Amer. Math. Soc. (N.S.) 27 (1992), no. 1, 1-67, DOI 10.1090/S0273-0979-199200266-5. MR1118699

[CL83] Michael G. Crandall and Pierre-Louis Lions, Viscosity solutions of Hamilton-Jacobi equations, Trans. Amer. Math. Soc. 277 (1983), no. 1, 1-42, DOI 10.2307/1999343. MR690039

[Eva80] Lawrence C. Evans, On solving certain nonlinear partial differential equations by accretive operator methods, Israel J. Math. 36 (1980), no. 3-4, 225-247, DOI 10.1007/BF02762047. MR597451

[FS06] Wendell H. Fleming and H. Mete Soner, Controlled Markov processes and viscosity solutions, 2nd ed., Stochastic Modelling and Applied Probability, vol. 25, Springer, New York, 2006. MR2179357

[GNT08] Wilfrid Gangbo, Truyen Nguyen, and Adrian Tudorascu, Hamilton-Jacobi equations in the Wasserstein space, Methods Appl. Anal. 15 (2008), no. 2, 155-183, DOI 10.4310/MAA.2008.v15.n2.a4. MR2481677

[Ham28] William R. Hamilton, Theory of systems of rays, Trans. Roy. Irish Acad. 15 (1828), 69-174.

[Hyn20] Ryan Hynd, A trajectory map for the pressureless Euler equations, Trans. Amer. Math. Soc. 373 (2020), no. 10, 6777-6815, DOI 10.1090/tran/8118. MR4155191

[HIP20] Ryan Hynd, Dennis Ikpe, and Terrance Pendleton, An eradication time problem for the SIR model, 2020.
[HK15] Ryan Hynd and Hwa Kil Kim, Value functions in the Wasserstein spaces: finite time horizons, J. Funct. Anal. 269 (2015), no. 4, 968-997, DOI 10.1016/j.jfa.2015.05.011. MR3352761

[Isa65] Rufus Isaacs, Differential games. A mathematical theory with applications to warfare and pursuit, control and optimization, John Wiley \& Sons, Inc., New York-London-Sydney, 1965. MR0210469

[Jac84] Carl Gustav Jacob Jacobi, Vorlesungen über Dynamik. Gehalten an der Universität zu Königsberg im Wintersemester 1842-1843 und nach einem von C. W. Borchart ausgearbeiteten hefte. hrsg. von A. Clebsch, 1884 (accessed February 20, 2021) https://catalog.hathitrust.org /Record/000163907.

[LL07] Jean-Michel Lasry and Pierre-Louis Lions, Mean field games, Jpn. J. Math. 2 (2007), no. 1, 229-260, DOI 10.1007/s11537-007-0657-8. MR2295621

[NXY09] James Nolen, Jack Xin, and Yifeng Yu, Bounds on front speeds for inviscid and viscous G-equations, Methods Appl. Anal. 16 (2009), no. 4, 507-520, DOI 10.4310/MAA.2009.v16.n4.a6, MR2734499

[Pet00] Norbert Peters, Turbulent combustion, Cambridge Monographs on Mechanics, Cambridge University Press, Cambridge, 2000, DOI 10.1017/CBO9780511612701. MR1792350

[Tra21] H. V. Tran, Hamilton-Jacobi equations: Theory and applications, Graduate Studies in Mathematics, vol. 213, American Mathematical Society, Providence, RI, 2021.

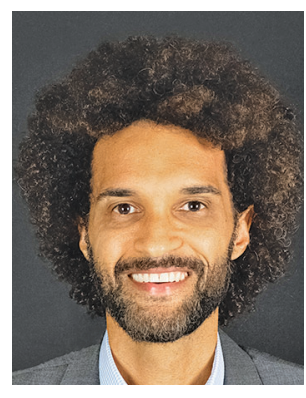

Ryan Hynd

Credits

Opening image is courtesy of nevarpp via Getty.

Figures 1-11 are courtesy of Ryan Hynd.

Author photo is courtesy of Nicholaus J. Booth. 